Research Article

\title{
Pinning Adaptive Synchronization of Delayed Coupled Dynamical Networks via Periodically Intermittent Control
}

\author{
Xueliang Liu ${ }^{1}$ and Shengbing $\mathrm{Xu}^{2}$ \\ ${ }^{1}$ School of Electronic Engineering and Intelligentization, Dongguan University of Technology, Dongguan 523000, China \\ ${ }^{2}$ City College, Dongguan University of Technology, Dongguan 523000, China
}

Correspondence should be addressed to Shengbing Xu; xusb2010@126.com

Received 9 April 2017; Accepted 30 August 2017; Published 9 October 2017

Academic Editor: R. Aguilar-López

Copyright (C) 2017 Xueliang Liu and Shengbing Xu. This is an open access article distributed under the Creative Commons Attribution License, which permits unrestricted use, distribution, and reproduction in any medium, provided the original work is properly cited.

\begin{abstract}
This paper investigates the exponential synchronization problem of delayed coupled dynamical networks by using adaptive pinning periodically intermittent control. Based on the Lyapunov method, by designing adaptive feedback controller, some sufficient conditions are presented to ensure the exponential synchronization of coupled dynamical networks with delayed coupling. Furthermore, a numerical example is given to demonstrate the validity of the theoretical results.
\end{abstract}

\section{Introduction}

Complex networks have received a great deal of attention due to their many potential practical applications $[1,2]$. A family of dynamically interacting units composes a kind of complex networks which can exhibit a number of emerging phenomena. Among various dynamical behaviors of complex networks, synchronization is a significant and interesting phenomenon, such as synchronization phenomena on the Internet, synchronization transfer of digital or analog signals in communication network, and synchronization related to biological neural networks. Recently, much works have been devoted to research the synchronization problem of complex networks [3-5].

In the case where the network cannot synchronize by itself, in order to drive the network to synchronize, many effective control techniques have been reported, such as feedback control [6], sampled-data control [7], adaptive control $[8,9]$, pinning control [10], impulsive control [11], and intermittent control [12]. In [9], the synchronization of a class of complex network by adding an adaptive controller to all nodes has been discussed. But in practice, it is too costly and impractical to add controllers to all nodes in a largescale network. To reduce the number of controlled nodes, pinning control is introduced [10], in which controllers are only applied to partial nodes. This case of control techniques has been earlier reported in paper [11-14]. In addition, the adaptive pinning control method, which is utilized to get the appropriate control gains effectively, has received considerable research attention. An adaptive pinning control method is proposed in [15] to synchronize for a delayed complex dynamical network with free coupling matrix. Besides these, there are many literatures to study adaptive pinning control problems of networks [16-18].

One the other hand, intermittent control has been widely used in engineering fields due to its practical and easy implementation in engineering control. In recent years, many important and interesting results on stabilization and synchronization of delayed dynamical networks by using intermittent control have been obtained. Based on $\infty$-norm, authors in [19] investigated a class of Cohen-Grossberg neural networks with time-varying delays by designing a periodically intermittent controller. In [20], by using periodically intermittent control, Gan studied the stochastic neural networks with leakage delay and reaction-diffusion terms; some new and less conservative synchronization conditions based on $p$-norm were derived. The pinning periodically intermittent control is used to achieve the synchronization of delayed complex network [21, 22]. To the best of our knowledge, the problem of adaptive pinning synchronization 
for delayed coupled dynamical networks has received very little research attention.

In this paper, we aim to further investigate adaptive pinning synchronization of delayed coupled dynamical network via periodically intermittent control. By using Lyapunov stability theory and designing adaptive feedback control gains, several criteria are given to guarantee synchronization of delayed coupled dynamical networks. A numerical simulation is also presented to show the effectiveness of the proposed method.

\section{Model and Preliminaries}

Consider the complex network consisting of $N$ nodes and the $i$ th node described by the following state equation:

$$
\begin{aligned}
\dot{x}_{i}(t)= & C x_{i}(t)+A f\left(x_{i}(t)\right)+B g\left(x_{i}\left(t-\tau_{1}\right)\right) \\
& +\sum_{j=1}^{N} g_{i j} \Gamma x_{j}(t)+\sum_{j=1}^{N} \widehat{g}_{i j} \Gamma_{\tau} x_{j}\left(t-\tau_{2}\right)+u_{i},
\end{aligned}
$$

where $x_{i}(t)=\left[x_{i 1}(t), x_{i 2}(t), \ldots, x_{i n}(t)\right]^{T} \in R^{n}$ is the state variable of node $i$ at time $t ; C=\left(c_{i j}\right)_{n \times n}, A=\left(a_{i j}\right)_{n \times n}$, and $B=\left(b_{i j}\right)_{n \times n}$ are system matrices, $f\left(x_{i}\right)=\left[f_{1}\left(x_{i 1}\right), f_{2}\left(x_{i 2}\right)\right.$, $\left.\ldots, f_{n}\left(x_{i n}\right)\right]^{T}$ and $g\left(x_{i}\right)=\left[g_{1}\left(x_{i 1}\right), g_{2}\left(x_{i 2}\right), \ldots, g_{n}\left(x_{i n}\right)\right]^{T}$ are continuous vector functions, and $\tau_{1}$ is the internal delay. $\Gamma=$ $\operatorname{diag}\left(d_{1}, d_{2}, \ldots, d_{n}\right)$ and $\Gamma_{\tau}=\operatorname{diag}\left(d_{1}^{\tau}, d_{2}^{\tau}, \ldots, d_{n}^{\tau}\right)$ are inner coupling matrices between the connected nodes $i$ and $j$ at time $t$ and $t-\tau_{2}$, where $\tau_{2}$ is the transmittal delay. $G=$ $\left(g_{i j}\right)_{N \times N}$ and $\widehat{G}=\left(\widehat{g}_{i j}\right)_{N \times N}$ are the configuration matrices; if there is a link from node $i$ to node $j$ at time $t$ (at time $t-\tau_{2}$ ), then $g_{i j}>0\left(\widehat{g}_{i j}>0\right)$, where $j \neq i$. Otherwise, $g_{i j}=0\left(\widehat{g}_{i j}=\right.$ $0)$. It is assumed that $G$ and $\widehat{G}$ satisfy the diffusive coupling connection, $\sum_{j=1}^{N} g_{i j}=0$ and $\sum_{j=1}^{N} \widehat{g}_{i j}=0 . u_{i} \in R^{n}$ are the control inputs. Note that the coupling configuration matrix $G$ and matrices $A, B, C, \Gamma$ and $\Gamma_{\tau}$ are not assumed to be symmetric.

The initial conditions of (1) are given by $x_{i}(t)=x_{i}^{0}(t)$, $t \in\left[t_{0}-\tau, t_{0}\right]$, and $x_{i}^{0}(t) \in C\left(\left[t_{0}-\tau, t_{0}\right], R^{n}\right)$, where $\tau=$ $\max \left(\tau_{1}, \tau_{2}\right)$. To discuss global synchronization with one delay coupling, we define the set

$$
S=\left\{\left[x_{1}^{T}, x_{2}^{T}, \ldots, x_{N}^{T}\right]^{T} \mid x_{i}=x_{j}, i, j=1,2, \ldots, N\right\}
$$

as the synchronization manifold for network (1). For all $s(t) \epsilon$ $S$, the dynamical equation of $s(t)$ satisfies

$$
\dot{s}(t)=C s(t)+A f(s(t))+B g\left(s\left(t-\tau_{1}\right)\right) .
$$

Define error states as $e_{i}(t)=x_{i}(t)-s(t)(1 \leq i \leq N)$. Then, we can derive the following error dynamical system:

$$
\begin{aligned}
\dot{e}_{i}(t)= & C e_{i}(t)+A\left(f\left(x_{i}(t)\right)-f(s(t))\right) \\
& +B\left(g\left(x_{i}\left(t-\tau_{1}\right)\right)-g\left(s\left(t-\tau_{1}\right)\right)\right) \\
& +\sum_{j=1}^{N} g_{i j} \Gamma e_{j}(t)+\sum_{j=1}^{N} g_{i j} \Gamma_{\tau} e_{j}\left(t-\tau_{2}\right)+u_{i}, \\
i=1,2, \ldots, N . &
\end{aligned}
$$

For convenience of statements, one has following assumption.

$H_{1}$ : There exists positive constants $l_{i}, l_{i}^{\prime}, i=1,2, \ldots, n$, for any $x, y \in R$, such that

$$
\begin{aligned}
& \left|f_{i}(x)-f_{i}(y)\right|<l_{i}|x-y|, \quad i=1,2, \ldots, n \\
& \left|g_{i}(x)-g_{i}(y)\right|<l_{i}^{\prime}|x-y|, \quad i=1,2, \ldots, n
\end{aligned}
$$

In order to derive the main results, the following definitions and lemmas are needed in this paper.

Lemma 1 (see [20]). Let $w:[\mu-\tau,+\infty) \rightarrow[0,+\infty)$ be a continuous function such that

$$
\dot{w}(t) \leq-a w(t)+b \max w_{t}
$$

is satisfied for $t \geq \mu$. If $a>b>0$; then

$$
w(t) \leq\left[\max w_{\mu}\right] e^{-\varepsilon(t-\mu)}, \quad t \geq \mu,
$$

where $\max w_{t}=\sup _{t-\tau \leq s \leq t} w(s)$ and $\varepsilon>0$ is the smallest real root of the equation

$$
\varepsilon-a+b e^{\varepsilon \tau}=0
$$

Lemma 2 (see [20]). Let $w:[\mu-\tau,+\infty) \rightarrow[0,+\infty)$ be $a$ continuous function such that

$$
\dot{w}(t) \leq a w(t)+b \max w_{t}
$$

is satisfied for $t \geq \mu$. If $a>0, b>0$, then

$$
w(t) \leq \max w_{t} \leq\left[\max w_{\mu}\right] e^{(a+b)(t-\mu)}, \quad t \geq \mu,
$$

where $\max w_{t}=\sup _{t-\tau \leq s \leq t} w(s)$.

Lemma 3 (see [6]). Let $x, y \in R^{n}$. Then

$$
2 x^{T} y \leq \epsilon x^{T} x+\epsilon^{-1} y^{T} y,
$$

for any $\epsilon>0$.

\section{Main Result}

In order to realize synchronization of the couple network by pinning periodically intermittent control, some controllers are added to selected partial nodes, and the controllers $u_{i}(1 \leq$ $i \leq N)$ can be described by

$$
u_{i}= \begin{cases}-\bar{k}_{i}(t) \Gamma e_{i}(t), & t \in[n T,(n+\theta) T) \\ 0, & t \in[(n+\theta) T,(n+1) T),\end{cases}
$$

where $T>0$ denotes the control period, $0<\theta<1, n \in \mathcal{N}$ :

$$
\bar{k}_{i}(t)= \begin{cases}k_{i}(t), & 1 \leq i \leq l, \\ 0, & l+1 \leq i \leq N,\end{cases}
$$


and $k_{i}(t)$ is the adaptive feedback strength for which the update law is to be designed. When $t \in[n T,(n+\theta) T)$ the error system (4) can be rewritten as

$$
\begin{aligned}
\dot{e}_{i}(t)= & C e_{i}(t)+A\left(f\left(x_{i}(t)\right)-f(s(t))\right) \\
& +B\left(g\left(x_{i}\left(t-\tau_{1}\right)\right)-g\left(s\left(t-\tau_{1}\right)\right)\right) \\
& +\sum_{j=1}^{N} g_{i j} \Gamma e_{j}(t) \\
& +\sum_{j=1}^{N} \widehat{g}_{i j} \Gamma_{\tau} e_{j}\left(\mathrm{t}-\tau_{2}\right)-\bar{k}_{i}(t) \Gamma e_{i}(t), \\
& \quad i=1,2, \ldots, N .
\end{aligned}
$$

When $t \in[(n+\theta) T,(n+1) T)$, the error system (4) can be rewritten as

$$
\begin{aligned}
\dot{e}_{i}(t)= & C e_{i}(t)+A\left(f\left(x_{i}(t)\right)-f(s(t))\right) \\
& +B\left(g\left(x_{i}\left(t-\tau_{1}\right)\right)-g\left(s\left(t-\tau_{1}\right)\right)\right) \\
& +\sum_{j=1}^{N} g_{i j} \Gamma e_{j}(t)+\sum_{j=1}^{N} \widehat{g}_{i j} \Gamma_{\tau} e_{j}\left(t-\tau_{2}\right), \\
& \quad i=1,2, \ldots, N .
\end{aligned}
$$

Our objective is to design suitable $T$ and $\theta$ such that the delayed couple network can realize synchronization. The main results are stated as follows.

Theorem 4. Suppose that Hypothesis $H_{1}$ holds, $\tau \leq \theta T$ and $\tau \leq(1-\theta) T$, where $\tau=\max \left\{\tau_{1}, \tau_{2}\right\}$. If there exist positive constants $\eta, a_{i}, r_{i}, \varepsilon_{i},(i=1,2)$, such that

(i) $\Pi_{1}=\left[\begin{array}{cccc}C^{s}+\eta I_{n} & \varepsilon_{1} A & L & \varepsilon_{2} B \\ \varepsilon_{1} A^{T} & -2 \varepsilon_{1} I_{n} & 0 & 0 \\ L & 0 & -2 \varepsilon_{1} I_{n} & 0 \\ \varepsilon_{2} B^{T} & 0 & 0 & -2 \varepsilon_{2} I_{n}\end{array}\right] \leq 0$,

(ii) $\Pi_{2}=\left[\begin{array}{cc}\left(-\eta+a_{1}\right) I_{N}+d_{j} G^{s}-K^{*} & (1 / 2) d_{j}^{\tau} \widehat{G} \\ (1 / 2) d_{j}^{\tau} \widehat{G}^{T} & -r_{1} I_{N}\end{array}\right] \leq 0$,

(iii) $\Pi_{3}=\left[\begin{array}{cc}\left(-\eta-a_{2}\right) I_{N}+d_{j} G^{s} & (1 / 2) d_{j}^{\tau} \widehat{G} \\ (1 / 2) d_{j}^{\tau} \widetilde{G}^{T} & -r_{2} I_{N}\end{array}\right] \leq 0$,

(iv) $a_{1}>r_{1}+q$,

(v) $\omega=\varepsilon-2\left(a_{1}+a_{2}+\left(r_{2}-r_{1}\right) e^{\varepsilon \tau}\right)(1-\theta)>0$,

where $C^{s}=(1 / 2)\left(C+C^{T}\right), K=\operatorname{diag}\left(k_{1}^{*}, \ldots, k_{l}^{*}, 0, \ldots, 0\right), q=$ $\max \left\{l_{i}^{\prime 2} / 2 \varepsilon_{2}, i=1, \ldots, n\right\}$, and $\varepsilon>0$ is the unique positive solution of the equation $\varepsilon-2 a_{1}+2(r+q) e^{\varepsilon \tau}=0$, and choosing the adaptive law

$$
\dot{k}_{i}(t)=\theta_{i} e_{i}^{T}(t) \Gamma e_{i}(t), \quad i=1,2, \ldots, l,
$$

then the controlled couple network (1) is globally exponentially synchronized.

Proof. Construct the following Lyapunov function:

$$
V(t)=\frac{1}{2} \sum_{i=1}^{N} e_{i}^{T}(t) e_{i}(t)+\frac{1}{2} \sum_{i=1}^{l} \frac{1}{\theta_{i}} \widetilde{k}_{i}^{2}
$$

where $\widetilde{k}_{i}=k_{i}(t)-k_{i}^{*}$ and $k_{i}^{*}, i=1,2, \ldots, l$, are positive constants.

Then the derivative of $V(t)$ with respect to time $t$ along the solutions of (14) and (15) can be calculated as follows: when $n T \leq t<(n+\theta) T$, for $n=0,1,2, \ldots$, we get

$$
\begin{aligned}
& \dot{V}(t)=\sum_{i=1}^{N} e_{i}^{T}(t)\left\{C e_{i}(t)+A\left(f\left(x_{i}(t)\right)-f(s(t))\right)\right. \\
& +B\left(g\left(x_{i}\left(t-\tau_{1}\right)\right)-g\left(s\left(t-\tau_{1}\right)\right)\right)+\sum_{j=1}^{N} g_{i j} \Gamma e_{j}(t) \\
& \left.+\sum_{j=1}^{N} \widehat{g}_{i j} \Gamma_{\tau} e_{j}\left(t-\tau_{2}\right)-\bar{k}_{i}(t) \Gamma e_{i}(t)\right\}+\sum_{i=1}^{l} \frac{1}{\theta_{i}}\left(k_{i}(t)\right. \\
& \left.-k_{i}^{*}\right) \dot{k}_{i}(t)=\sum_{i=1}^{N} e_{i}^{T}(t)\left\{C e_{i}(t)\right. \\
& +A\left(f\left(x_{i}(t)\right)-f(s(t))\right) \\
& +B\left(g\left(x_{i}\left(t-\tau_{1}\right)\right)-g\left(s\left(t-\tau_{1}\right)\right)\right)+\sum_{j=1}^{N} g_{i j} \Gamma e_{j}(t) \\
& \left.+\sum_{j=1}^{N} \widehat{g}_{i j} \Gamma_{\tau} e_{j}\left(t-\tau_{2}\right)\right\}-\sum_{i=1}^{l} k_{i}(t) e_{i}^{T}(t) \Gamma e_{i}(t) \\
& +\sum_{i=1}^{l} \frac{1}{\theta_{i}}\left(k_{i}(t)-k_{i}^{*}\right) \dot{k}_{i}(t) .
\end{aligned}
$$

Based on Lemma 3, we have

$$
\begin{aligned}
& e_{i}^{T}(t) A\left(f\left(x_{i}(t)\right)-f(s(t))\right) \\
& \leq \frac{\varepsilon_{1}}{2} e_{i}^{T}(t) A A^{T} e_{i}^{T}(t) \\
&+\frac{1}{2 \varepsilon_{1}}\left\|f\left(x_{i}(t)\right)-f(s(t))\right\|^{2} \\
& \leq \frac{1}{2} e_{i}^{T}(t)\left(\varepsilon_{1} A A^{T}+\frac{1}{\varepsilon_{1}} L L\right) e_{i}(t), \\
& e_{i}^{T}(t) B\left(g\left(x_{i}\left(t-\tau_{1}\right)\right)-g\left(s\left(t-\tau_{1}\right)\right)\right) \\
& \leq \frac{\varepsilon_{2}}{2} e_{i}^{T}(t) B B^{T} e_{i}^{T}(t) \\
& \quad+\frac{1}{2 \varepsilon_{2}}\left\|g\left(x_{i}\left(t-\tau_{1}\right)\right)-g\left(s\left(t-\tau_{1}\right)\right)\right\|^{2} \\
& \leq \frac{\varepsilon_{2}}{2} e_{i}^{T}(t) B B^{T} e_{i}(t) \\
& \quad+\frac{1}{2 \varepsilon_{2}} e_{i}^{T}\left(t-\tau_{1}\right) L^{\prime} L^{\prime} e_{i}\left(t-\tau_{1}\right),
\end{aligned}
$$

where $L=\operatorname{diag}\left(l_{1}, l_{2}, \ldots, l_{n}\right)$, and $L^{\prime}=\operatorname{diag}\left(l_{1}^{\prime}, l_{2}^{\prime}, \ldots, l_{n}^{\prime}\right)$. 
Substituting (16) into the following expression, one has

$$
\begin{aligned}
\sum_{i=1}^{l} & \frac{1}{\theta_{i}}\left(k_{i}(t)-k_{i}^{*}\right) \dot{k}_{i}(t)-\sum_{i=1}^{l} k_{i}(t) e_{i}^{T}(t) \Gamma e_{i}(t) \\
& =-\sum_{i=1}^{l} k_{i}^{*} e_{i}^{T}(t) \Gamma e_{i}(t) .
\end{aligned}
$$

Using exchange of rows and columns, it is easy to get

$$
\sum_{i=1}^{N} \sum_{j=1}^{N} e_{i}^{T}(t) g_{i j} \Gamma e_{j}(t)=\sum_{j=1}^{n} d_{j} \tilde{e}_{i}^{T}(t) G \widetilde{e}_{j}(t)
$$

where $\tilde{e}_{j}(t)=\left[e_{1 j}(t), e_{2 j}(t), \ldots, e_{N j}(t)\right]^{T}$.

By following the similar steps denoted in (22), we get that

$$
\begin{aligned}
\sum_{i=1}^{N} \sum_{j=1}^{N} \widehat{g}_{i j} e_{i}^{T}(t) \Gamma_{\tau} e_{j}\left(t-\tau_{2}\right) & =\sum_{j=1}^{n} d_{j}^{\tau} \widetilde{e}_{j}^{T}(t) \widehat{G}^{\tilde{e}_{j}}\left(t-\tau_{2}\right) \\
\sum_{i=1}^{l} k_{i}^{*} e_{i}^{T}(t) \Gamma e_{i}(t) & =\sum_{j=1}^{n} d_{j} \tilde{e}_{j}^{T}(t) K^{*} \widetilde{e}_{j}(t),
\end{aligned}
$$

where $K^{*}=\operatorname{diag}\left(k_{1}^{*}, k_{2}^{*}, \ldots, k_{l}^{*}, 0, \ldots, 0\right)$.

Substituting (19)-(23) into (18), we have

$\dot{V}(t)$

$$
\begin{aligned}
\leq & \sum_{i=1}^{N} e_{i}^{T}(t)\left(C+\frac{1}{2} \varepsilon_{1} A A^{T}+\frac{1}{2 \varepsilon_{1}} L L+\frac{\varepsilon_{2}}{2} B B^{T}\right) e_{i}(t) \\
& +\sum_{j=1}^{n} \widetilde{e}_{j}^{T}(t)\left[d_{j}(G-K) \widetilde{e}_{j}(t)+d_{j}^{\tau} \widehat{G}_{j}\left(t-\tau_{2}\right)\right] \\
& +\frac{1}{2 \varepsilon_{2}} \sum_{i=1}^{N} e_{i}^{T}\left(t-\tau_{1}\right) L^{\prime} L^{\prime} e_{i}\left(t-\tau_{1}\right) .
\end{aligned}
$$

Define $q=\max \left\{l_{i}^{\prime 2} / 2 \varepsilon_{2}, i=1,2, \ldots, n\right\}$, and based on conditions (i) and (ii) of Theorem 4 , one gets

$$
\begin{aligned}
& \dot{V}(t) \leq \sum_{i=1}^{N}\left(-a_{1} e_{i}^{T}(t) e_{i}(t)+q e_{i}^{T}\left(t-\tau_{1}\right) e_{i}\left(t-\tau_{1}\right)\right. \\
& \left.+r_{1} e_{i}^{T}\left(t-\tau_{2}\right) e_{i}\left(t-\tau_{2}\right)\right) \leq-2 a_{1} V(t)+2\left(q+r_{1}\right) \\
& \quad \sup _{t-\tau \leq s \leq t} V(s) .
\end{aligned}
$$

Similarly, based on condition (i) and (iii) of Theorem 4, when $(n+\theta) T \leq t \leq(n+1) T$, we have

$\dot{V}(t)$

$$
\leq \sum_{i=1}^{N} e_{i}^{T}(t)\left(C+\frac{1}{2} \varepsilon_{1} A A^{T}+\frac{1}{2 \varepsilon_{1}} L L+\frac{\varepsilon_{2}}{2} B B^{T}\right) e_{i}(t)
$$

$$
\begin{gathered}
+\sum_{j=1}^{n} \widetilde{e}_{j}^{T}(t)\left[d_{j} G \widetilde{e}_{j}(t)+d_{j}^{\tau} \widehat{G} \widetilde{e}_{j}\left(t-\tau_{2}\right)\right] \\
+\frac{1}{2 \varepsilon_{2}} \sum_{i=1}^{N} e_{i}^{T}\left(t-\tau_{1}\right) L^{\prime} L^{\prime} e_{i}\left(t-\tau_{1}\right) \\
\leq 2 a_{2} V(t)+2\left(q+r_{2}\right) \sup _{t-\tau \leq s \leq t} V(s) .
\end{gathered}
$$

In the following, we will prove that conditions (iv) and (v) imply

$$
V(t)=\sup _{-\tau \leq s \leq 0} V(s) e^{-\omega t}, \quad t \geq 0
$$

Take $M_{0}=\max _{-\tau \leq s \leq 0} V(s)$ and $W(t)=e^{\varepsilon t} V(t)$. For $0 \leq$ $t \leq \theta T$, based on (25) and by using Lemma 1 , we obtain

$$
V(t) \leq \max _{-\tau \leq s \leq 0} V(s) e^{-\varepsilon t},
$$

where $\varepsilon>0$ is the smallest real root of the equation

$$
\varepsilon-2 a_{1}+2\left(q+r_{1}\right) e^{\varepsilon \tau}=0 .
$$

Thus, we have

$$
W(t) \leq M_{0}
$$

For $\theta T \leq t \leq T$, based on (26), we have

$\dot{W}(t) \leq \varepsilon W(t)$

$$
\begin{gathered}
+2 e^{\varepsilon t}\left(a_{2} V(t)+\left(r_{2}+q\right) \sup _{t-\tau \leq s \leq t} V(s)\right) \\
\leq\left(\varepsilon+2 a_{2}\right) W(t)+2\left(r_{2}+q\right) e^{\varepsilon \tau} \sup _{t-\tau \leq s \leq t} W(s) .
\end{gathered}
$$

From Lemma 2 , and noting that $\varepsilon-2 a_{1}+2\left(q+r_{1}\right) e^{\varepsilon \tau}=0$, we obtain

$$
\begin{aligned}
W(t) & \leq \sup _{\theta T-\tau \leq s \leq \theta T} W(s) e^{\left(\varepsilon+2 a_{2}+2\left(r_{2}+q\right) e^{\varepsilon \tau}\right)(t-\theta T)} \\
& \leq M_{0} e^{2\left(a_{1}+a_{2}+\left(r_{2}-r_{1}\right) e^{\varepsilon \tau}\right)(t-\theta T)} \\
& \leq M_{0} e^{2\left(a_{1}+a_{2}+\left(r_{2}-r_{1}\right) e^{\varepsilon \tau}\right)(1-\theta) t} .
\end{aligned}
$$

For $T \leq t<(1+\theta) T$, based on (25) and by using Lemma 1, we obtain

$$
V(t) \leq \sup _{T-\tau \leq s \leq T} V(s) e^{-\varepsilon(t-T)} .
$$

Consider that $2\left(a_{1}+a_{2}+\left(r_{2}-r_{1}\right) e^{\varepsilon \tau}\right)-\varepsilon>0$; then

$$
\begin{aligned}
W(t) & \leq \sup _{T-\tau \leq s \leq T} W(s) e^{\varepsilon(T-s)} \\
& \leq \sup _{T-\tau \leq s \leq T} M_{0} e^{2\left(a_{1}+a_{2}+\left(r_{2}-r_{1}\right) e^{\varepsilon \tau}\right)(s-\theta T)-\varepsilon s+\varepsilon T} \\
& \leq M_{0} e^{2\left(a_{1}+a_{2}+\left(r_{2}-r_{1}\right) e^{\varepsilon \tau}\right)(1-\theta) T} \\
& \leq M_{0} e^{2\left(a_{1}+a_{2}+\left(r_{2}-r_{1}\right) e^{\varepsilon \tau}\right)(1-\theta) t}
\end{aligned}
$$


For $(1+\theta) T \leq t<2 T$, one can repeat the same argument and get similar results to (31) and (32). It can be deduced from Lemma 2 that

$W(t)$

$$
\begin{aligned}
& \leq \sup _{(1+\theta) T-\tau \leq s \leq(1+\theta) T} W(s) e^{\left(\varepsilon+2\left(r_{2}+q\right) e^{\varepsilon \tau}+2 a_{2}\right)(t-(1+\theta) T)} \\
& \leq M_{0} e^{2\left(a_{1}+a_{2}+\left(r_{2}-r_{1}\right) e^{\varepsilon \tau}\right)(1-\theta) t} .
\end{aligned}
$$

By induction, we can derive the following estimation of $W(t)$ for any integer $n$. For $n T \leq t<(n+1) T$

$$
W(t) \leq M_{0} e^{2\left(a_{1}+a_{2}+\left(r_{2}-r_{1}\right) e^{\varepsilon \tau}\right)(1-\theta) t} .
$$

Form the definition of $W(t)$ and condition (v) of Theorem 4, we obtain

$$
\begin{aligned}
V(t) \leq M_{0} e^{-\left(\varepsilon-2\left(a_{1}+a_{2}+\left(r_{2}-r_{1}\right) e^{\varepsilon \tau}\right)(1-\theta)\right) t}=M_{0} e^{-\omega t}, & \\
& t \geq 0 .
\end{aligned}
$$

This implies the conclusion and the proof is complete.

When $\Gamma=0$, only delayed coupling exists in the networks. One has the following corollary.

Corollary 5. Suppose that Hypothesis $H_{1}$ holds, $\tau \leq \theta T$ and $\tau \leq(1-\theta) T$ where $\tau=\tau_{2}$. If there exist positive constants $\eta, a_{i}, r_{i}, \varepsilon_{i},(i=1,2)$, such that

(i) $\Pi_{1}=\left[\begin{array}{cccc}C^{s}+\eta I_{n} & \varepsilon_{1} A & L & \varepsilon_{2} B \\ \varepsilon_{1} A^{T} & -2 \varepsilon_{1} I_{n} & 0 & 0 \\ L & 0 & -2 \varepsilon_{1} I_{n} & 0 \\ \varepsilon_{2} B^{T} & 0 & 0 & -2 \varepsilon_{2} I_{n}\end{array}\right] \leq 0$,

(ii) $\Pi_{2}=\left[\begin{array}{cc}\left(-\eta+a_{1}\right) I_{N}-K^{*} & (1 / 2) d_{j}^{\tau} \widehat{G} \\ (1 / 2) d_{j}^{\tau} \widehat{G}^{T} & -r_{1} I_{N}\end{array}\right] \leq 0$,

(iii) $\Pi_{3}=\left[\begin{array}{ll}\left(-\eta-a_{2}\right) I_{N} & (1 / 2) d_{j}^{\tau} \widehat{G} \\ (1 / 2) d_{j}^{\tau} \widetilde{G}^{T} & -r_{2} I_{N}\end{array}\right] \leq 0$,

(iv) $a_{1}>r_{1}+q$,

(v) $\omega=\varepsilon-2\left(a_{1}+a_{2}+\left(r_{2}-r_{1}\right) e^{\varepsilon \tau}\right)(1-\theta)>0$,

where $K=\operatorname{diag}\left(k_{1}^{*}, \ldots, k_{l}^{*}, 0, \ldots, 0\right), q=\max \left\{l_{i}^{\prime 2} / 2 \varepsilon_{2}, i=\right.$ $1, \ldots, n\}$, and $\varepsilon>0$ is the unique positive solution of the equation $\varepsilon-2 a_{1}+2(r+q) e^{\varepsilon \tau}=0$, and choosing the adaptive law as (16), then the controlled couple network (1) is globally exponentially synchronized.

When $\Gamma_{\tau}=0$, there is no delayed coupling in the coupled networks.

Corollary 6. Suppose that Hypothesis $H_{1}$ holds, $\tau \leq \theta T$ and $\tau \leq(1-\theta) T$ where $\tau=\tau_{1}$. If there exist positive constants $\eta, a_{i}, r_{i}, \varepsilon_{i},(i=1,2)$, such that

(i) $\Pi_{1}=\left[\begin{array}{cccc}C^{s}+\eta I_{n} & \varepsilon_{1} A & L & \varepsilon_{2} B \\ \varepsilon_{1} A^{T} & -2 \varepsilon_{1} I_{n} & 0 & 0 \\ L & 0 & -2 \varepsilon_{1} I_{n} & 0 \\ \varepsilon_{2} B^{T} & 0 & 0 & -2 \varepsilon_{2} I_{n}\end{array}\right] \leq 0$,

(ii) $\Pi_{2}=\left(-\eta+a_{1}\right) I_{N}+d_{j} G^{s}-K^{*} \leq 0$,

(iii) $\Pi_{3}=\left(-\eta-a_{2}\right) I_{N}+d_{j} \widehat{G}^{s} \leq 0$, (iv) $a_{1}>q=\max \left\{l_{i}^{\prime 2} / 2 \varepsilon_{2}, i=1, \ldots, n\right\}$,

(v) $\omega=\varepsilon-2\left(a_{1}+a_{2}\right)(1-\theta)>0$,

where $K=\operatorname{diag}\left(k_{1}^{*}, \ldots, k_{l}^{*}, 0, \ldots, 0\right)$ and $\varepsilon>0$ is the unique positive solution of the equation $\varepsilon-2 a_{1}+2(r+q) e^{\varepsilon \tau}=0$, and choosing the adaptive law as (16), then the controlled couple network (1) is globally exponentially synchronized.

\section{Numerical Simulation}

In this section, we present a numerical simulation to illustrate the feasibility and effectiveness of our results.

Consider the coupled network (1) consisting of 6 identical Chua oscillators with time delayed nonlinearity. The dynamics of the Chua oscillator is given by

$$
\dot{x}_{i}(t)=C x_{i}(t)+A f\left(x_{i}(t)\right)+B g\left(x_{i}\left(t-\tau_{1}\right)\right),
$$

where $x_{i}(t) \in R^{3}, \tau_{1}=0.02, f\left(x_{i}(t)\right)=(-(1 / 2) \delta(\alpha-$ $\left.\beta)\left(\left|x_{i 1}(t)+1\right|-\left|x_{i 1}(t)-1\right|\right), 0,0\right)^{T}$, and $g\left(x_{i}\left(t-\tau_{1}\right)\right)=$ $\left(-\xi \gamma \sin \left(v x_{i 1}\left(t-\tau_{1}\right)\right), 0,0\right)^{T}$ :

$$
\begin{aligned}
& C=\left[\begin{array}{ccc}
-\delta(1+\beta) & \delta & 0 \\
1 & -1 & 1 \\
0 & -\xi & -\epsilon
\end{array}\right], \\
& A=\left[\begin{array}{lll}
1 & 0 & 0 \\
0 & 0 & 0 \\
0 & 0 & 0
\end{array}\right], \\
& B=\left[\begin{array}{lll}
0 & 0 & 0 \\
0 & 0 & 0 \\
1 & 0 & 0
\end{array}\right], \\
& \delta=10 \text {, } \\
& \epsilon=0.1636 \text {, } \\
& \xi=19.53, \\
& \alpha=-1.4325, \\
& \beta=-0.7831 \text {, } \\
& v=0.5 \text {, } \\
& \gamma=0.2 \text {; }
\end{aligned}
$$

the other parameters of network are given as follows:

$$
\begin{aligned}
\Gamma & =2 I_{2}, \\
\Gamma_{\tau} & =0.1 I_{2}, \\
\tau_{2} & =0.1,
\end{aligned}
$$




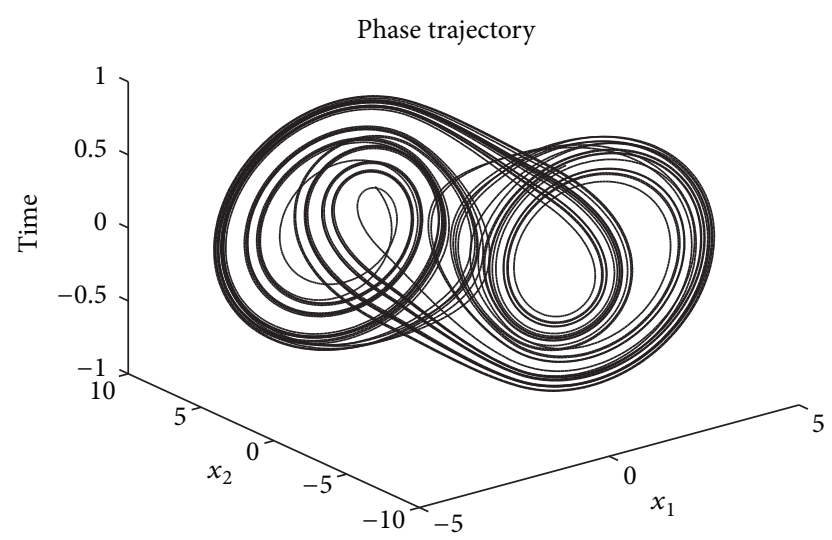

Figure 1: Dynamical behavior of the synchronization manifold $s(t)$.

$$
\begin{aligned}
& G=10\left[\begin{array}{cccccc}
-5 & 1 & 1 & 1 & 1 & 1 \\
1 & -5 & 1 & 1 & 1 & 1 \\
1 & 1 & -5 & 1 & 1 & 1 \\
1 & 1 & 1 & -5 & 1 & 1 \\
1 & 1 & 1 & 1 & -5 & 1 \\
1 & 1 & 1 & 1 & 1 & -5
\end{array}\right], \\
& \widehat{G}=\left[\begin{array}{ccccccc}
-2 & 1 & 1 & 0 & 0 & 0 \\
1 & -2 & 1 & 0 & 0 & 0 & \\
0 & 1 & -2 & 1 & 0 & 0 \\
0 & 0 & 1 & -2 & 1 & 0 \\
0 & 0 & 0 & 1 & -2 & 1 \\
0 & 0 & 0 & 1 & 1 & -2
\end{array}\right] .
\end{aligned}
$$

The dynamical behavior of the synchronization manifold $s(t)$ is shown in Figure 1.

It is easy to verify that nonlinear functions $f(\cdot)$ and $g(\cdot)$ satisfy the Lipschitz condition with $l_{1}=(1 / 2) \delta(\beta-\alpha), l_{2}=$ $\xi \gamma \nu$. By using the MATLAB LMI toolbox, a feasible solution of condition (i) of Theorem 4 is obtained as follows:

$$
\begin{gathered}
\varepsilon_{1}=6.5041, \\
\varepsilon_{2}=2.0684, \\
\eta=-12.56 .
\end{gathered}
$$

In this simulation, we add the adaptive feedback controllers to the 1st, 2nd, and 3rd nodes. Let $T=1, \theta=0.2, \omega=$ $0.1, a_{1}=20, a_{2}=13, r_{1}=2, r_{2}=0.2$, and $K^{*}=$ $\operatorname{diag}(500,500,500,0,0,0)$; thus the condition in Theorem 4 is satisfied. According to Theorem 4, the coupled network (1) can be achieved pinning adaptive synchronization.

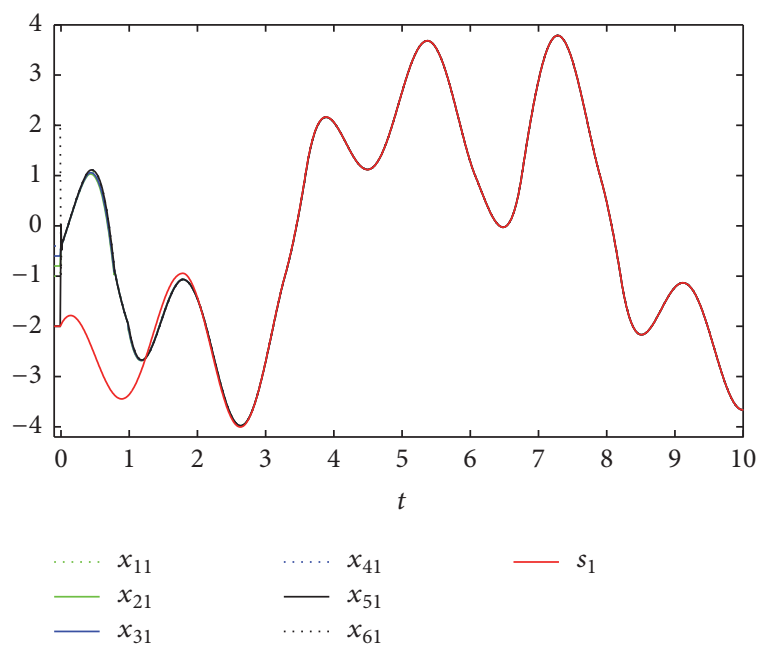

FIGURE 2: The trajectories of the state variables of $x_{i 1}(t),(i=$ $1,2, \ldots, 6)$ in the controlled network (1) and synchronization manifold $s_{1}(t)$.

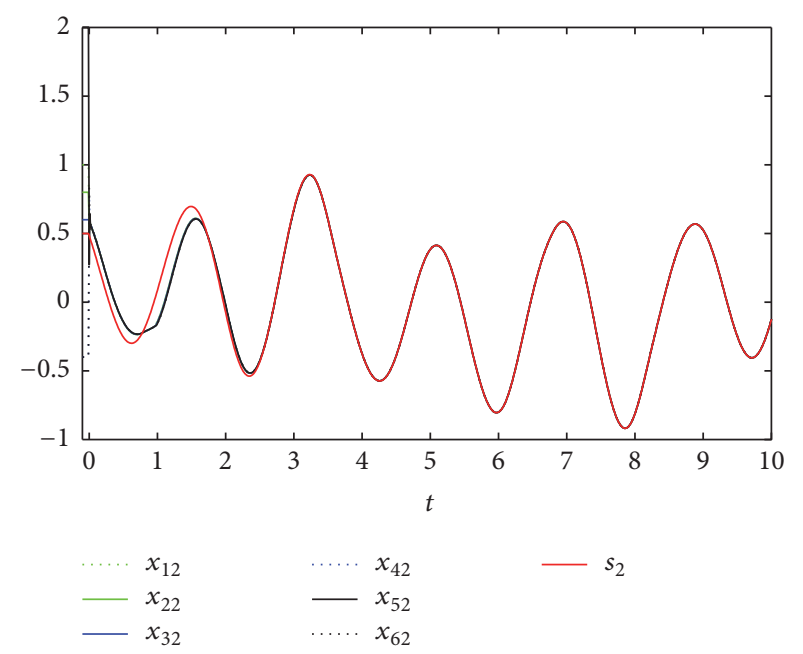

FIgURE 3: The trajectories of the state variables of $x_{i 2}(t), \quad(i=1,2$, $\ldots, 6)$ in the controlled network (1) and synchronization manifold $s_{2}(t)$.

The initial conditions of the numerical simulation are as follows:

$$
\begin{aligned}
X^{0} & =\left[\begin{array}{lllllll}
x_{i}^{0}(t) & x_{2}^{0}(t) & \cdots & x_{6}^{0}(t) & s(t)
\end{array}\right] \\
& =\left[\begin{array}{ccccccc}
-1 & -0.8 & -0.6 & -0.4 & -2 & 2 & -2 \\
1 & 0.8 & 0.6 & -0.4 & 2 & -0.4 & 0.5 \\
0.5 & 0.3 & 1 & -2 & -0.4 & 0.4 & 1
\end{array}\right] .
\end{aligned}
$$

The simulation results are given in Figures $2-5$. The trajectories of the state variables $x_{i}(t)$ and synchronization manifold are illustrated in Figures $2-4$. From these figures, we can see that all nodes $x_{i}(t)$ tend to the synchronization manifold $s(t)$. The time evolution of the feedback control gains $\bar{k}_{i}(t)(i=1,2,3)$ is shown in Figure 5 . 


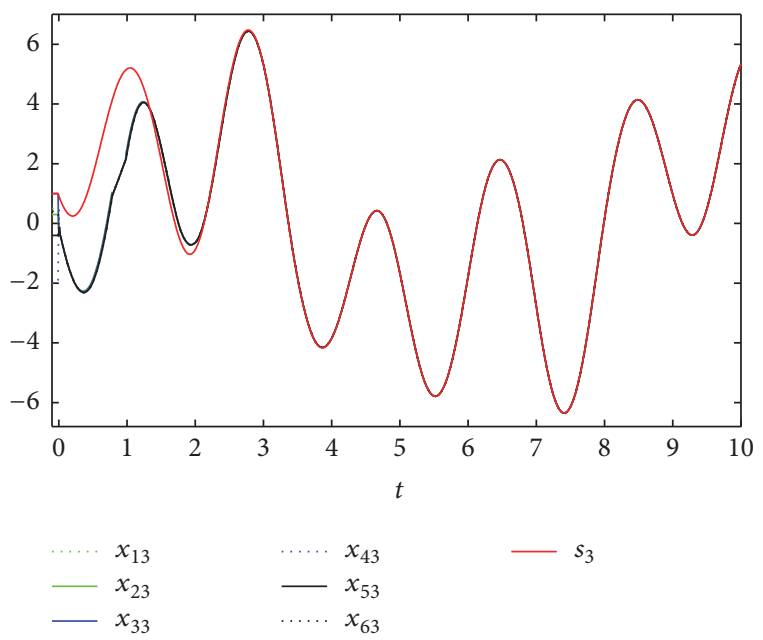

FIgURE 4: The trajectories of the state variables of $x_{i 2}(t), \quad(i=1,2$, $\ldots, 6)$ in the controlled network (1) and synchronization manifold $s_{3}(t)$.
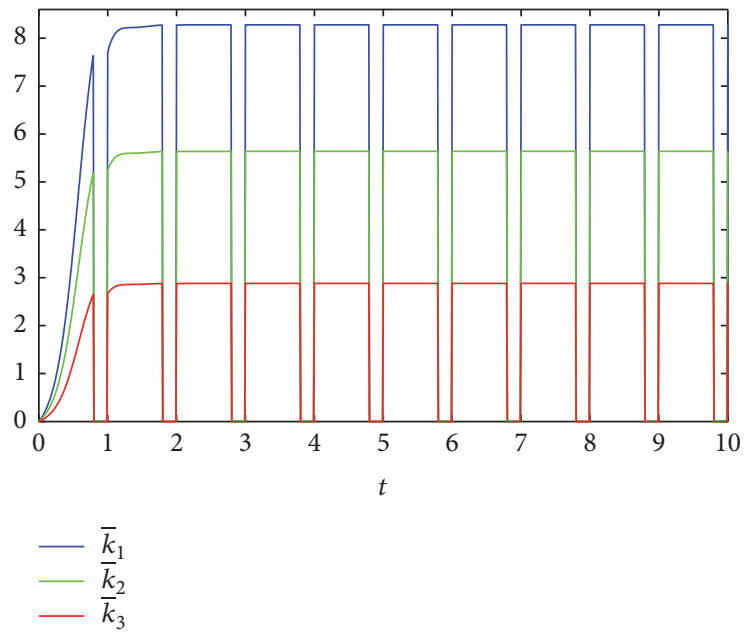

FIgURE 5: Time evolution of the control gains $\bar{k}_{1}, \bar{k}_{2}, \bar{k}_{3}$.

\section{Conclusion}

In this paper, we have investigated the exponential synchronization problem for neural networks by pinning periodically intermittent control. Based on Lyapunov stability theory and periodically intermittent control method, some novel conditions for synchronization are derived. Furthermore, numerical simulations have verified the effectiveness of the presented method.

\section{Conflicts of Interest}

The authors declare that they have no conflicts of interest.

\section{Authors' Contributions}

Xueliang Liu and Shengbing Xu conceived and designed the study. Xueliang Liu carried out the main part of this manuscript. Shengbing Xu participated in the discussion and corrected the main theorem. All authors read and approved the manuscript.

\section{Acknowledgments}

This work is supported by the Fund of Natural Science Foundation of Guangdong Province (2014A030310418) and the Science and Technology Planning Project of Guangdong Province (2014A050503068).

\section{References}

[1] S. Boccaletti, V. Latora, Y. Moreno, M. Chavez, and D.-U. Hwang, "Complex networks: structure and dynamics," Physics Reports. A Review Section of Physics Letters, vol. 424, no. 4-5, pp. 175-308, 2006.

[2] S. H. Strogatz, "Exploring complex networks," Nature, vol. 410, no. 6825, pp. 268-276, 2001.

[3] X. Liu and T. Chen, "Synchronization analysis for nonlinearlycoupled complex networks with an asymmetrical coupling matrix," Physica A, vol. 387, no. 16-17, pp. 4429-4439, 2008.

[4] W. Wu and T. Chen, "Global synchronization criteria of linearly coupled neural network systems with time-varying coupling," IEEE Transactions on Neural Networks, vol. 19, no. 2, pp. 319$332,2008$.

[5] W. Zhou, A. Dai, J. Yang, H. Liu, and X. Liu, "Exponential synchronization of Markovian jumping complex dynamical networks with randomly occurring parameter uncertainties," Nonlinear Dynamics. An International Journal of Nonlinear Dynamics and Chaos in Engineering Systems, vol. 78, no. 1, pp. 15-27, 2014.

[6] Q. Wei, X.-y. Wang, and X.-p. Hu, "Chaos synchronization in complex oscillators networks with time delay via adaptive complex feedback control," Circuits, Systems, and Signal Processing, vol. 33, no. 8, pp. 2427-2447, 2014.

[7] B. Shen, Z. Wang, and X. Liu, "Sampled-data synchronization control of dynamical networks with stochastic sampling," Institute of Electrical and Electronics Engineers. Transactions on Automatic Control, vol. 57, no. 10, pp. 2644-2650, 2012.

[8] W. Zhou, D. Tong, Y. Gao, C. Ji, and H. Su, "Mode and delay-dependent adaptive exponential synchronization in pth moment for stochastic delayed neural networks with markovian switching," IEEE Transactions on Neural Networks \& Learning Systems, vol. 23, no. 4, pp. 662-668, 2012.

[9] J. Zhou, J. Lu, and J. Lu, "Adaptive synchronization of an uncertain complex dynamical network," Institute of Electrical and Electronics Engineers. Transactions on Automatic Control, vol. 51, no. 4, pp. 652-656, 2006.

[10] T. Chen, X. Liu, and W. Lu, "Pinning complex networks by a single controller," IEEE Transactions on Circuits and Systems. I. Regular Papers, vol. 54, no. 6, pp. 1317-1326, 2007.

[11] J. Lu, J. Kurths, J. Cao, N. Mahdavi, and C. Huang, "Synchronization control for nonlinear stochastic dynamical networks: Pinning impulsive strategy," IEEE Transactions on Neural Networks and Learning Systems, vol. 23, no. 2, pp. 285-292, 2012.

[12] W. Xia and J. Cao, "Pinning synchronization of delayed dynamical networks via periodically intermittent control," Chaos. An Interdisciplinary Journal of Nonlinear Science, vol. 19, no. 1, article 013120, 2009. 
[13] Q. Song and J. Cao, "On pinning synchronization of directed and undirected complex dynamical networks," IEEE Transactions on Circuits and Systems. I. Regular Papers, vol. 57, no. 3, pp. 672-680, 2010.

[14] Q. Song, J. Cao, and F. Liu, "Pinning synchronization of linearly coupled delayed neural networks," Mathematics and Computers in Simulation, vol. 86, pp. 39-51, 2012.

[15] T. H. Lee, J. H. Park, H. Y. Jung, S. M. Lee, and O. M. Kwon, "Synchronization of a delayed complex dynamical network with free coupling matrix," Nonlinear Dynamics. An International Journal of Nonlinear Dynamics and Chaos in Engineering Systems, vol. 69, no. 3, pp. 1081-1090, 2012.

[16] X. Gong and Z. Wu, "Adaptive pinning impulsive synchronization of dynamical networks with time-varying delay," Advances in Difference Equations, vol. 240, 2015.

[17] F. Wang, Y. Yang, M. Hu, and X. Xu, "Projective cluster synchronization of fractional-order coupled-delay complex network via adaptive pinning control," Physica A. Statistical Mechanics and its Applications, vol. 434, pp. 134-143, 2015.

[18] X. Sun, Z. Feng, and X. Liu, "Pinning adaptive synchronization of neutral-type coupled neural networks with stochastic perturbation," Advances in Difference Equations, vol. 77, 2014.

[19] J. Yu, C. Hu, H. Jiang, and Z. Teng, "Exponential synchronization of Cohen-Grossberg neural networks via periodically intermittent control," Neurocomputing, vol. 74, no. 10, pp. 17761782, 2011.

[20] Q. Gan, "Exponential synchronization of stochastic neural networks with leakage delay and reaction-diffusion terms via periodically intermittent control," Chaos. An Interdisciplinary Journal of Nonlinear Science, vol. 22, no. 1, article 013124, 2012.

[21] W. Xia and J. Cao, "Pinning synchronization of delayed dynamical networks via periodically intermittent control," Chaos. An Interdisciplinary Journal of Nonlinear Science, vol. 19, no. 1, article 013120, Article ID 013120, 2009.

[22] S. Cai, J. Hao, Q. He, and Z. Liu, "Exponential synchronization of complex delayed dynamical networks via pinning periodically intermittent control," Physics Letters, Section A: General, Atomic and Solid State Physics, vol. 375, no. 19, pp. 1965-1971, 2011. 


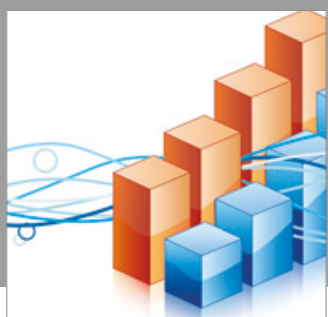

Advances in

Operations Research

vatersals

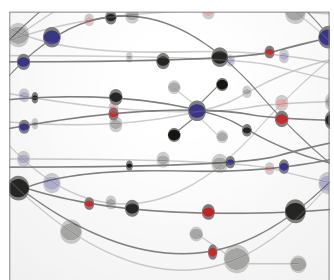

\section{The Scientific} World Journal
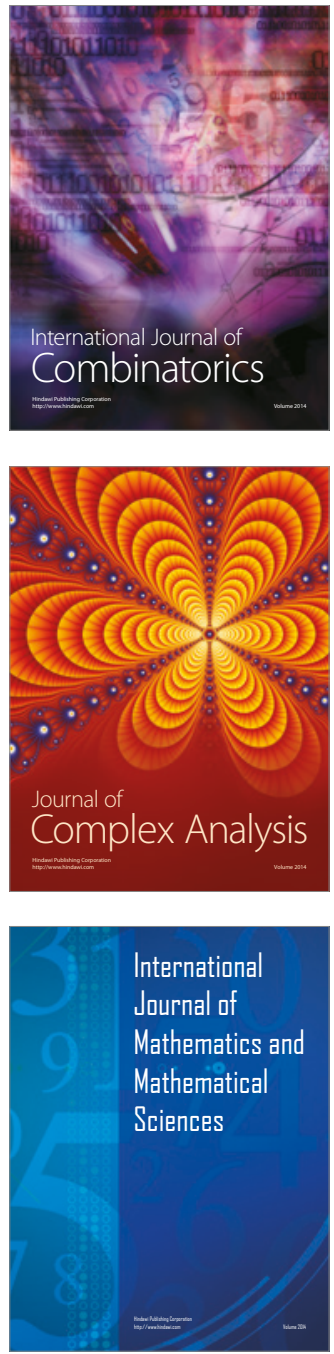
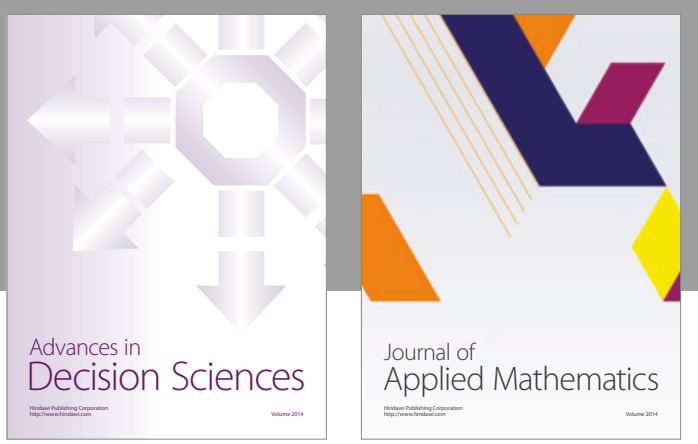

Algebra

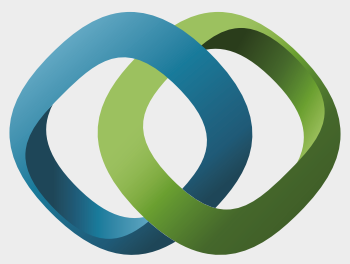

\section{Hindawi}

Submit your manuscripts at

https://www.hindawi.com
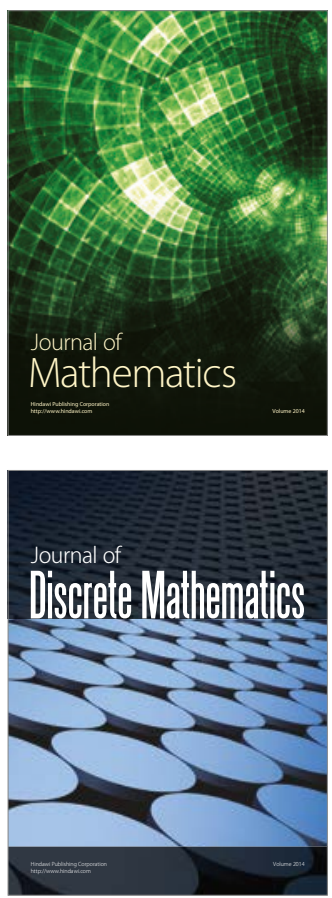

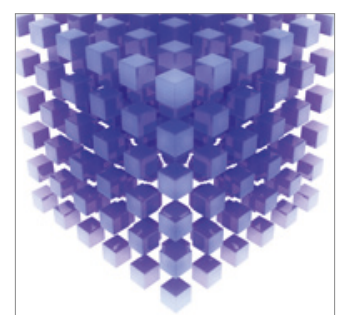

Mathematical Problems in Engineering
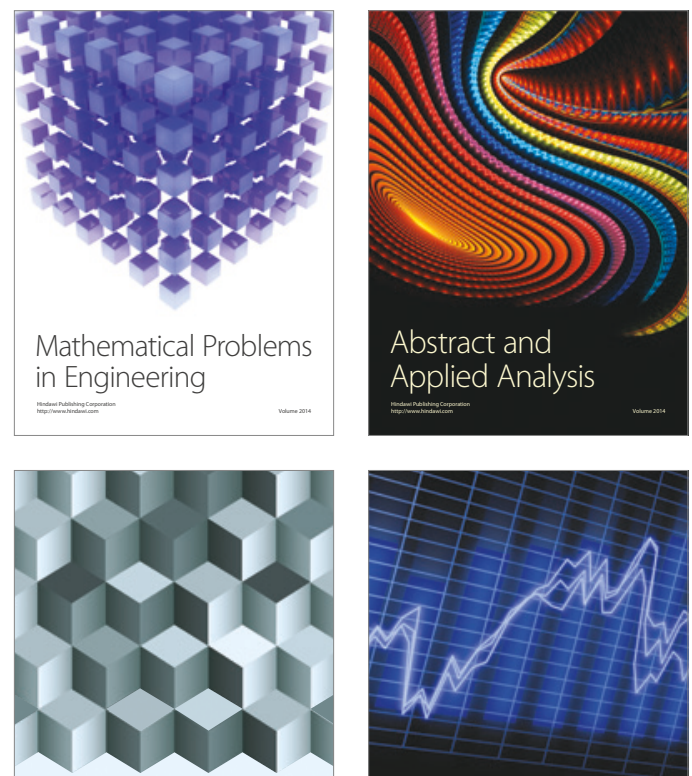

Journal of

Function Spaces

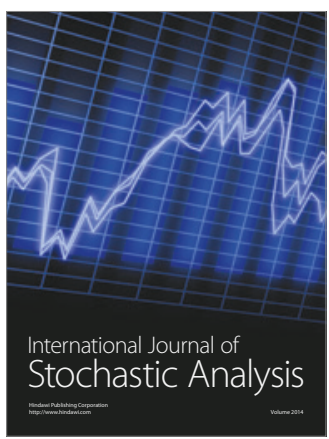

Probability and Statistics
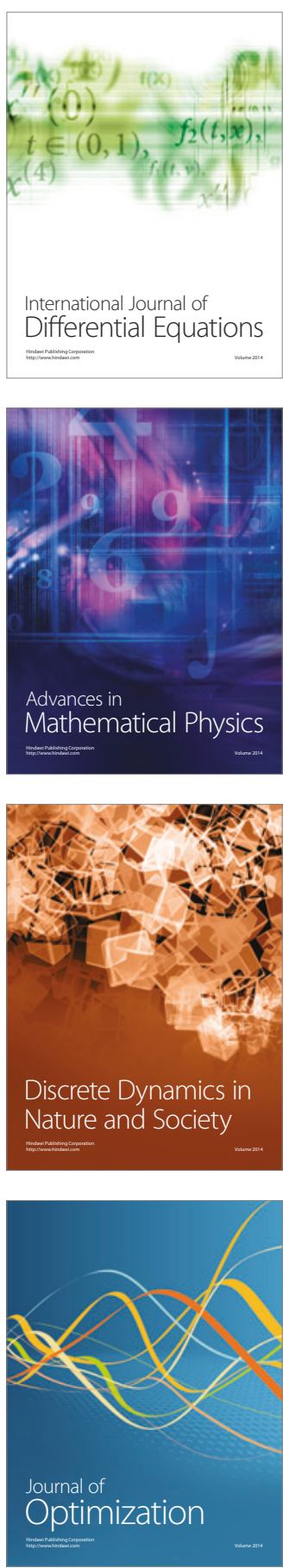